\title{
Mineração
}

\section{Aplicação do Índice Global de Sustentabilidade na explotação de coquina na península de Santa Elena}

\author{
Wilmer José Vásquez Granda \\ Mestrando do Programa de Pós-Graduação em Engenharia Mineral \\ Departamento de Engenharia de Minas/EM/UFOP \\ E-mail:wvasques@demin.ufop.br
}

Hernani Mota de Lima

Professor Adjunto do Departamento de Engenharia de Minas/EM/UFOP

E-mail:hernani.lima@ufop.br

\section{Resumo}

Na Península de Santa Elena (Equador), desenvolvem-se, paralelamente, a explotação artesanal e a mecanizada da rocha coquina. Para estabelecer a sustentabilidade da explotação, foram estabelecidos indicadores, suficientemente flexíveis à realidade local e de fácil aplicação. Para determinação do quão sustentável é a explotação de coquina, usou-se o Índice Global de Sustentabilidade (IGS). Esse estudo apresenta os resultados do IGS aplicado ao caso da explotação artesanal no Morro Tablazo.

Palavras-chave: Indicadores de sustentabilidade, pequena mineração.

\begin{abstract}
In Santa Elena's Peninsula (Ecuador), the artisanal and mechanical mining of coquina rock work in parallel. In order to establish the sustainability of the exploitation, indicators were chosen that were sufficiently flexible for local reality and easy to apply. To determine how sustainable the coquina exploitation is, the Global Sustainability Index (GSI) was used. This study presents the GSI results for the Morro Tablazo artisanal exploitation case.
\end{abstract}

Keywords: sustainability indicators, small mining operations. 


\section{Introdução}

A Península de Santa Elena, localizada no centro-oeste do território equatoriano, é caracterizada por um clima desértico. A economia local baseia-se no turismo, pesca e, nos últimos anos, a agricultura tem demonstrado crescimento significativo depois da transposição das águas do Rio Daule. Na região, a mineração, em pequena escala (artesanal e mecanizada) de coquina (calcário detrítico), no Morro Tablazo, constitui uma importante fonte de matérias para a construção civil, que, também, tem experimentado um acréscimo considerável impulsionado pelo aumento do turismo.

Esse estudo descreve a utilização de indicadores de sustentabilidade para explotação de coquina na península de Santa Elena, os quais podem ser utilizados como instrumentos políticos de apoio à pequena mineração na região, bem como instrumentos de gestão ambiental por parte das empresas de modo a garantir o desenvolvimento econômico da região e a preservação do meio ambiente.

Para qualificar e quantificar a sustentabilidade sócio-econômica e ambiental da explotação de coquina no Morro Tablazo, fez-se necessário determinar indicadores que pudessem ser facilmente mensuráveis e aplicáveis à realidade local. Para tal, utilizou-se o Índice Global de Sustentabilidade (IGS).

\section{Indicador de sustentabilidade}

Muitos sistemas trabalham com base em fatores internos ou externos, que podem afetar positiva ou negativamente a sustentabilidade de um empreendimento. Indicadores são medidas que servem de base para avaliar o estado de qualquer sistema, mediante uma forma clara de interpretação, bem como para diagnosticar as alternativas de gestão de um empreendimento.

Em mineração, os indicadores constituem uma espécie de guia de reconhecimento dos recursos existentes, das opções, de suas vantagens, comercialização, relações com o ambiente natural e interação com os meios sociais. Todos esses parâmetros são governados por indicadores específicos, que dão a conhecer o impacto generalizado da atividade mineira sobre o meio ambiente e a comunidade. Os indicadores devem ter as seguintes características fundamentais:

- Fácil medição.

- Aplicabilidade em diferentes ecossistemas e sistemas econômicos e sociais.

- Amplitude.

- Praticidade e facilidade de entendimento.

- Repetitividade em relação ao tempo.

- Adaptabilidade e sensibilidade às mudanças no sistema.

- Tolerância aos diversos padrões estabelecidos.

Um indicador de sustentabilidade é uma categoria de indicador ambiental usado na medição de parâmetros de uma dimensão ambiental dentro de uma estratégia de desenvolvimento sustentável (EEA, 2002). Na indústria extrativa são conhecidos muitos indicadores, essencialmente de impacto ambiental, porém apenas alguns são definidos como indicadores de sustentabilidade, sendo apenas usados aqueles que podem ser quantificados.

O indicador de sustentabilidade, proposto nesse estudo, utiliza, como metodologia, a realização de uma avaliação de sustentabilidade para uma atividade de mineração. Como resultado do teste, tem-se um indicador de sustentabilidade denominado de Índice Global de Sustentabilidade (IGS). Para obtenção deste, desenvolve-se uma "Checklist" com respostas sim ou não a uma série de questões, que, embora simples, são relativas à ações-chave da atividade de mineração (González \& Carvajal, 2002).

O IGS é definido pela expressão:

IGS $(\%)=\left\{\left[\Sigma_{\text {sim }}(C T+C A+C E L+C S C)\right] /\right.$ Total de ações $\}$ x 100

Onde:

CT é a Caracterização Técnica.

CAé a Caracterização Ambiental.

CEL é a Caracterização Econômica Legal.

CSC é a Caracterização Sócio-Cultural.

O resultado desse teste indica o nível de sustentabilidade da atividade mineira. Se o IGS é maior que 50\%, a atividade extrativa enquadra-se no campo da sustentabilidade e será muito mais sustentável quando mais se aproximar de 100\%. Se o IGS é menor que $50 \%$, ou igual a $50 \%$, a atividade extrativa é caracterizada como de não sustentabilidade e, portanto, deverá revisar todas as ações que leva a cabo.

A Tabela 1 apresenta os indicadores do teste de sustentabilidade propostos para o cálculo de IGS.

\section{Medida do IGS para a pequena mineração artesanal realizada pela Cooperativa Tablazo $\mathrm{N}^{\circ} 1$}

Os índices propostos na Tabela 1 para o cálculo do IGS foram modificados para atender à realidade local da pequena mineração artesanal realizada pela Cooperativa Tablazo N ${ }^{\circ} 1$ e são apresentados na Tabela 2. 
Wilmer José Vásquez Granda et al.

Tabela 1 - Indicadores de sustentabilidade para o cálculo de IGS.

\begin{tabular}{|c|c|c|c|}
\hline \multirow{2}{*}{$\begin{array}{l}\text { Nome do } \\
\text { indicador }\end{array}$} & \multirow[t]{2}{*}{ Tipo de ação } & \multicolumn{2}{|c|}{$\begin{array}{c}\text { Ação } \\
\text { executada }\end{array}$} \\
\hline & & SIM & NÃO \\
\hline \multirow{10}{*}{ 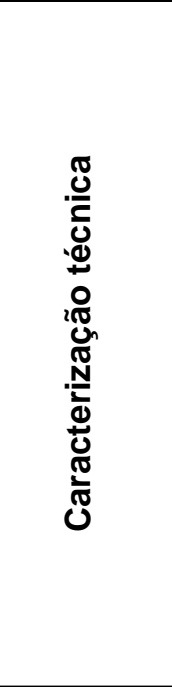 } & O background da área é conhecido? & & \\
\hline & Existem um plano de uso e administração das reservas? & & \\
\hline & Existe uma caracterização geomecânica para explotação? & & \\
\hline & Existe um sistema de manejo integral para as águas? & & \\
\hline & Existe um programa de prevenção de risco? & & \\
\hline & Existe um plano de ordenação territorial mineiro? & & \\
\hline & O método de explotação é otimizado? & & \\
\hline & Possui alguma certificação administrativa, por exemplo, ISO $9.000 ?$ & & \\
\hline & Existe um plano de fechamento de minas? & & \\
\hline & Existe algum plano de otimização da energia? & & \\
\hline \multirow{7}{*}{ 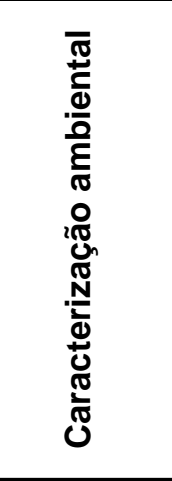 } & Existem um programa e controle de emissões? & & \\
\hline & Tem obtido alguma certificação ambiental, por exemplo, ISO $14.000 ?$ & & \\
\hline & Existe um estudo de impacto ambiental da explotação? & & \\
\hline & O plano de recuperação está concluído? & & \\
\hline & A companhia tem algum programa de auditoria ambiental? & & \\
\hline & O programa de minimização de resíduos está implementado? & & \\
\hline & Existe um plano de minimização de danos ambientais? & & \\
\hline \multirow{3}{*}{ 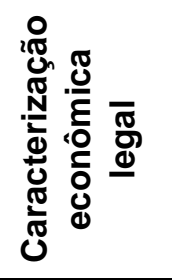 } & A Legislação ambiental é conhecida? & & \\
\hline & Existem compromissos administrativos, fiscais e de trabalho? & & \\
\hline & A companhia possui fundo de garantia de suas atividades? & & \\
\hline \multirow{6}{*}{ 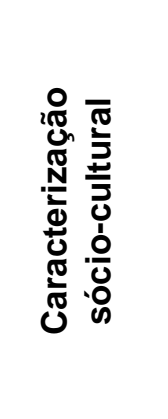 } & A companhia possui uma política informativa transparente? & & \\
\hline & Existe um compromisso e responsabilidade social da companhia? & & \\
\hline & Existem programas de formação continua e de treinamento para os trabalhadores? & & \\
\hline & Existem mecanismos de participação civil nas decisões da companhia? & & \\
\hline & Existe estudo de demanda social dos produtos mineiros? & & \\
\hline & Existe um estudo da repercussão da atividade mineira na população? & & \\
\hline & TOTAL & & \\
\hline
\end{tabular}

Fonte: González e Carvajal (2002). 
Aplicação do Índice Global de Sustentabilidade na explotação de coquina na península de Santa Elena Tabela 2 - Cálculo do IGS para a Cooperativa Tablazo Nº1 (modificada).

\begin{tabular}{|c|c|c|c|}
\hline \multirow{2}{*}{$\begin{array}{l}\text { Nome do } \\
\text { indicador }\end{array}$} & \multirow[t]{2}{*}{ Tipo de ação } & \multicolumn{2}{|c|}{$\begin{array}{c}\text { Ação } \\
\text { executada }\end{array}$} \\
\hline & & SIM & NÃO \\
\hline \multirow{6}{*}{ 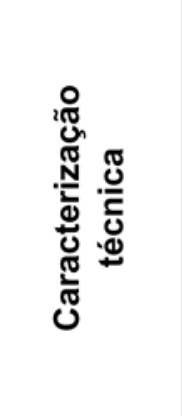 } & O background da área é conhecido? & & $\bullet$ \\
\hline & Existem um plano de uso e administração das reservas? & & $\bullet$ \\
\hline & Existe uma caracterização geomecânica para explotação? & & $\bullet$ \\
\hline & Existe um sistema de manejo integral para as águas? & & $\bullet$ \\
\hline & Existe um programa de prevenção de risco? & $\bullet$ & \\
\hline & O método de explotação é otimizado? & $\bullet$ & \\
\hline \multirow{5}{*}{ 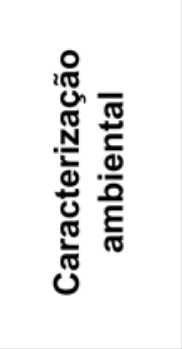 } & Existe um plano de fechamento de minas? & $\bullet$ & \\
\hline & Existe algum plano de otimização da energia? & NA & NA \\
\hline & Existem um programa e controle de emissões? & $\bullet$ & \\
\hline & Tem obtido alguma certificação ambiental, por exemplo, ISO $14.000 ?$ & NA & NA \\
\hline & Existe um estudo de impacto ambiental da explotação? & $\bullet$ & \\
\hline \multirow{3}{*}{ 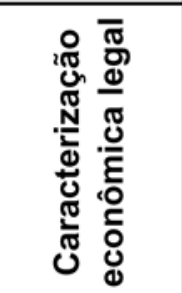 } & O plano de recuperação está concluído? & $\bullet$ & \\
\hline & A cooperativa tem algum programa de auditoria ambiental? & $\bullet$ & \\
\hline & O programa de minimização de resíduos está implementado? & & $\bullet$ \\
\hline \multirow{6}{*}{ 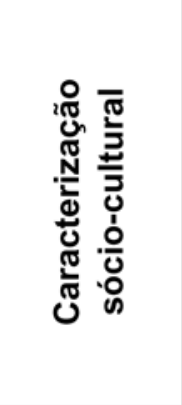 } & Existe um plano de minimização de danos ambientais? & $\bullet$ & \\
\hline & A Legislação ambiental é conhecida? & • & \\
\hline & Existem compromissos administrativos, fiscais e de trabalho? & & $\bullet$ \\
\hline & A cooperativa possui fundo de garantia de suas atividades? & $\bullet$ & \\
\hline & A cooperativa possui uma política informativa transparente? & $\bullet$ & \\
\hline & Existem um compromisso e responsabilidade social da cooperativa? & & $\bullet$ \\
\hline TOTAL & & 11 & \\
\hline
\end{tabular}

NA: Não Aplica

O cálculo do IGS, para a pequena mineração artesanal realizada pela Cooperativa Tablazo $N^{\circ} 1$, baseado na equação (1), é descrito a seguir:

$$
\begin{aligned}
& \operatorname{IGS}(\%)=\left\{\left[\sum_{\text {sim }}(\mathrm{CT}+\mathrm{CA}+\mathrm{CEL}+\mathrm{CSC})\right] / \text { Total de ações }\right\} \times 100 \\
& \left.\operatorname{IGS}(\%)=\left\{\left[\sum_{\text {sim }} 2+3+2+4\right)\right] / 18\right\} \times 100 \\
& \operatorname{IGS}(\%)=\{11 / 18\} \times 100 \\
& \operatorname{IGS}(\%)=61.11
\end{aligned}
$$

\section{Resultados e discussão}

O IGS calculado para a pequena mineração artesanal de coquina realizada pela Cooperativa Tablazo $\mathrm{N}^{\circ} 1$ foi de 61.11 e encontra-se no campo da sustentabilidade. 
Wilmer José Vásquez Granda et al.

O indicador de sustentabilidade calculado, no trabalho de dissertação (Granda, 2005), para a pequena mineração artesanal de coquina realizada pela Cooperativa Tablazo Nº 1 , foi de 78.63 e as áreas analisadas (técnica, ambiental, econômica legal, sócio-cultural) apresentam comportamento similar ao IGS.

Este IGS calculado é um valor típico das pequenas minerações artesanais, carentes de técnica, controle de impacto ambiental, assistência econômica legal e que apresenta problemas socio-econômicos e culturais.

Para melhorar a sustentabilidade da pequena mineração artesanal de coquina realizada pela Cooperativa Tablazo
$\mathrm{N}^{0} 1$, faz-se necessária a implementação de assistência na área técnica, principalmente, que é a que apresenta maior deficiência no teste de sustentabilidade.

O IGS é uma ferramenta de fácil aplicação, sobretudo em mineração artesanal pouco mecanizada, para estabelecer seu nível de sustentabilidade e brindar assistência nas áreas que apresentam deficiências no teste de sustentabilidade.

\section{Referências bibliográficas}

EEA. Información para mejorar el ambiente en Europa. In: Barcelona, lugar de encuentro de las dimensiones medioambiental, económica y social. Copenhague: European Environment Agency, 2002.

GRANDA, W. Mineração sustentável da rocha coquina na península de Santa Elena, Província do Guayas - Equador. Ouro Preto: Programa de Pós-Graduação em Engenharia Mineral. Departamento de Engenharia de Minas, Escola de Minas/UFOP, 2005. 118p. (Dissertação de Mestrado).

GONZÁLEZ, A., CARVAJAL, D. Sustainability Indicators in the Spanish Extractive Industry. In: Indicators of sustainability: for the mineral extraction industries. Rio de Janeiro: CNPq/CYTED, 2002. 409p.

Artigo recebido em 14/07/2005 e aprovado em 19/06/2006.

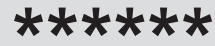

\section{REM - Revista Escola de Minas}

\section{0 anOS divulgando CIÊNCIA.}

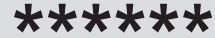

\section{REM: a mais antiga revista técnico- científica do setor mínero-metalúrgico.}

\author{
$* * * * * *$ \\ www.rem.com.br \\ *******
}

